\title{
Charmonium spectral functions in two-flavour QCD
}

G. Aarts ${ }^{\mathrm{a}}$, C. R. Allton ${ }^{\mathrm{a}}$, R. Morrin ${ }^{\mathrm{b}}$, A. Ó Cais ${ }^{\mathrm{b}}$, M. B. Oktay ${ }^{\mathrm{b}}$, M. J. Peardon ${ }^{\mathrm{b}}$, J. I. Skullerud ${ }^{\mathrm{b} *}$

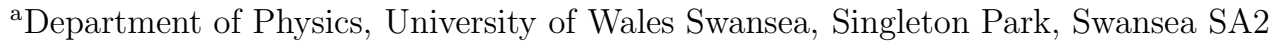
8PP, Wales, UK

${ }^{\mathrm{b}}$ School of Mathematics, Trinity College, Dublin 2, Ireland

We compute charmonium spectral functions in 2-flavour QCD using the maximum entropy method and anisotropic lattices. We find that the S-waves $\left(J / \psi\right.$ and $\left.\eta_{c}\right)$ survive up to temperatures close to $2 T_{c}$, while the P-waves $\left(\chi_{c 0}\right.$ and $\left.\chi_{c 1}\right)$ melt away below $1.3 T_{c}$.

\section{INTRODUCTION}

The fate of charmonium at high temperature has generated much interest the past 20 years [1]. Contrary to the original prediction that $J / \psi$ production would be highly suppressed immediately above the phase transition, recent theoretical and experimental results indicate a more complicated picture, where the $1 \mathrm{~S}$ states may survive up to high temperatures (possibly even with enhanced yields), while other states disappear earlier.

The properties of hadrons at high temperature are encoded in the spectral function $\rho_{\Gamma}$, which can be related to euclidean-time correlation functions $G_{\Gamma}$ as follows,

$G_{\Gamma}(\tau, \vec{p})=\int_{0}^{\infty} \frac{d \omega}{2 \pi} \rho_{\Gamma}(\omega, \vec{p}) \frac{\cosh [\omega(\tau-1 / 2 T)]}{\sinh (\omega / 2 T)}$

where the subscript $\Gamma$ correspond to the different quantum numbers. Determining the continuous function $\rho(\omega)$ from $G(\tau), \tau=1 \ldots L_{\tau}$, is an ill-posed problem, but it is possible to infer the most likely $\rho(\omega)$ using the maximum entropy method (MEM) [2]. This has been used to study spectral functions in the quenched approximation [3-5], but substantial uncertainties still remain.

In order to resolve some of these uncertainties, lattice simulations with dynamical fermions ( 2 or $2+1$ light flavours) would be highly desirable. However, in order to determine spectral functions using MEM, at least $\mathcal{O}(10)$ independent lattice points are needed in the imaginary time direction. At $T \sim 2 T_{c}$, this implies a temporal lattice spacing $a_{t} \lesssim 0.025 \mathrm{fm}$. If the spatial lattice spacing $a_{s}$ were to be the same, such a simulation would be far too expensive to carry out with current computing resources.

In order to make the simulation feasible, anisotropic lattices, with $a_{t} \ll a_{s}$, are therefore required. However, dynamical anisotropic lattice simulations introduce additional complications not present in isotropic or quenched anisotropic simulations. The anisotropic

\footnotetext{
*Speaker
} 
formulation introduces two additional parameters (the bare quark and gluon anisotropies), which must be tuned so that the physical anisotropies are the same for gauge and fermion fields. In the presence of dynamical fermions, this requires a simultaneous two-dimensional tuning, which has been described and carried out in Ref. [6].

We have previously presented preliminary results $[7,8]$, indicating that S-wave states survive well into the plasma phase. These simulations were carried out before the tuning was completed, and therefore had systematic uncertainties at the $20 \%$ level. Here we present results from a simulation where the anisotropies have been tuned to within $3 \%$, and study $\mathrm{P}$-waves in addition to $\mathrm{S}$-waves.

\section{RESULTS}

We use the TSI gauge action [9] and a coarse Wilson, fine Hamber-Wu fermion action [10] with stout-link smearing [11] as described in [6]. The spatial lattice spacing is $a_{s} \approx 0.18 \mathrm{fm}$ and the anisotropy $\xi=6$, while $m_{\pi} / m_{\rho}=0.54$; our simulation parameters correspond to run 6 in [6]. For the charm quarks we have used bare masses $a_{t} m=0.08$ and 0.092. The critical temperature was determined from studying the variation of the Polyakov loop as a function of $N_{\tau}$ on $12^{3} \times N_{\tau}$ lattices, giving $N_{\tau}^{c}=1 / a T_{c} \approx 34$. 500 configurations were generated on $8^{3} \times 32,8^{3} \times 24$ and $8^{3} \times 16$ lattices, corresponding to $T / T_{c} \approx 1.05,1.4$ and 2.1 respectively. Charmonium correlators were computed using all-to-all propagators [12] and analysed with Bryan's MEM algorithm [13] using the free continuum spectral function $\omega^{2}$ as default model.

Figure 1 show spectral functions for the pseudoscalar and vector channels, corresponding to the S-wave states $\eta_{c}$ and $J / \psi$ respectively. At the two lower temperatures $\left(N_{\tau}=32\right)$ and $N_{\tau}=24$ ) we see two peaks; however, the second (higher) peak is a lattice artefact which can be observed as a cusp in the free lattice spectral function [7]. The lower peak position corresponds to the S-wave ground state masses at zero temperature, indicating that these states survive more or less unchanged at least up to $1.4 T_{c}$. At $T \sim 2.1 T_{c}$ the picture is less clear: for the lighter mass $(m=0.08)$ the peak has disappeared in both channels; while for the heavier mass $(m=0.092)$ there is still a peak in the vector channel, but not in the pseudoscalar. To what extent these effects are genuine, i.e. whether they show a real mass dependence and the survival of the vector meson to higher temperatures than the pseudoscalar, cannot be ascertained at present.

In fig. 2 we show spectral functions for the scalar and axial-vector channels, corresponding to the $\mathrm{P}$-wave states $\chi_{c 0}$ and $\chi_{c 1}$ respectively. In the axial-vector channel there is a peak at $a_{t} \omega \approx 0.45$ for the lowest temperature, consistent with the presence of a modified $\chi_{c 1}$ at $1.05 T_{c}$. There is no discernible corresponding peak in the scalar channel. At the higher temperatures the peak has disappeared, indicating that these states have melted below $1.4 T_{c}$. The peak in the scalar spectral function at $a_{t} \omega \approx 0.25$ at $N_{\tau}=24$ is not clearly understood at present and requires further investigation.

\section{DISCUSSION}

The main conclusion that can be drawn from this study is that the $\mathrm{S}$-wave states $J / \psi$ and $\eta_{c}$ survive virtually unchanged in the medium above $T_{c}$, before melting at $T \lesssim 2 T_{c}$. The P-wave states, on the other hand, disappear shortly above the phase transition. 

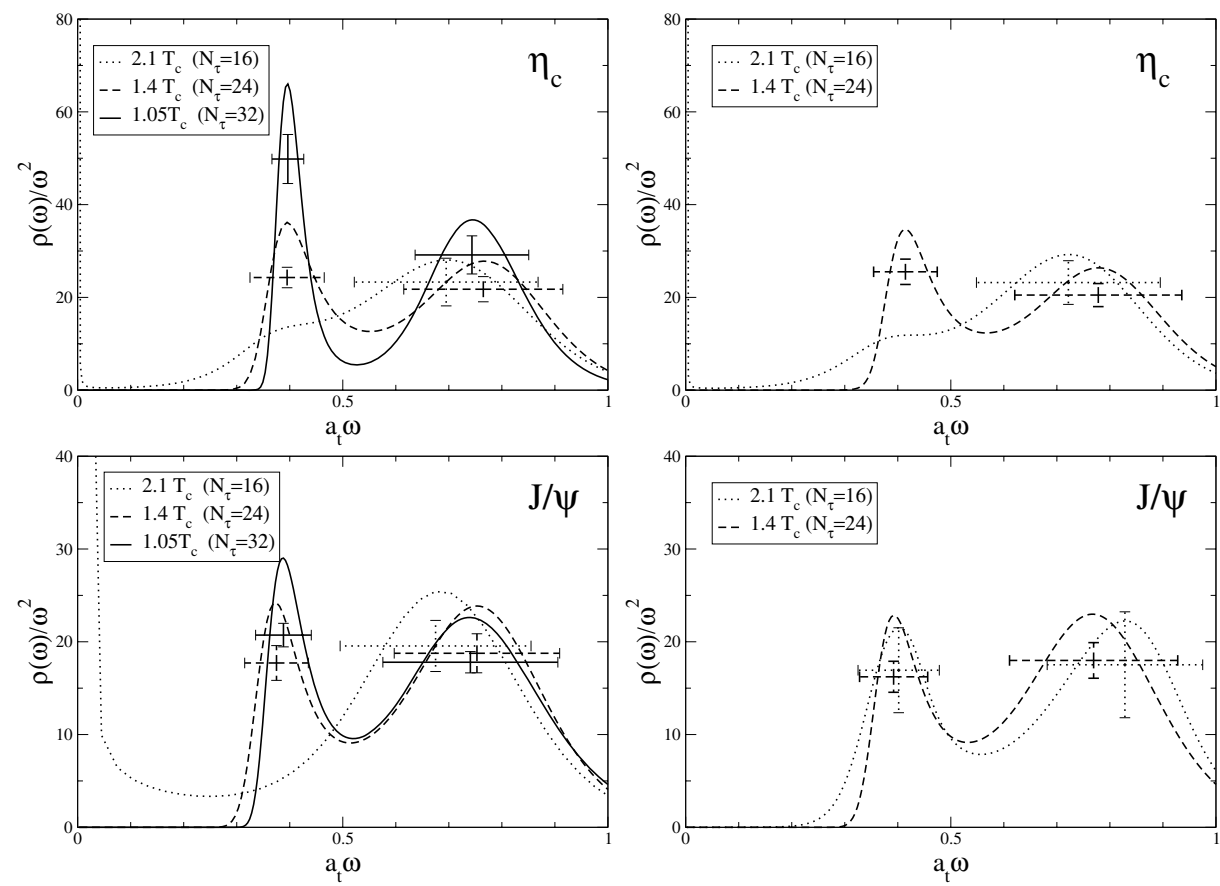

Figure 1. Spectral functions for the S-wave charmonium states $\eta_{c}$ (top) and $J / \psi$ (bottom), for $a_{t} m_{0}=0.080$ (left) and 0.092 (right).

Our results thus lend support to the qualitative picture that has emerged from quenched calculations. In order to make these results quantitative, a number of outstanding issues needs to be addressed:

- The $8^{3}$ lattice corresponds to a physical volume of $(1.4 \mathrm{fm})^{3}$, and finite volume effects are expected to be substantial, especially for the P-waves. We are currently repeating the calculation on $12^{3}$ lattices.

- A more detailed temperature scan will be necessary to determine the melting points of the different states. This is currently underway. A zero-temperature run is also in progress to provide a baseline for comparison.

- The present analysis has been carried out using only the free continuum spectral function as default model. In order to assess systematic uncertainties arising from the MEM, different default models will need to be employed. In particular, the free lattice spectral function [7] will provide a useful comparison.

In the final instance, simulations on finer lattices will be necessary to resolve the remaining uncertainties. This will however require a new nonperturbative tuning exercise, and 

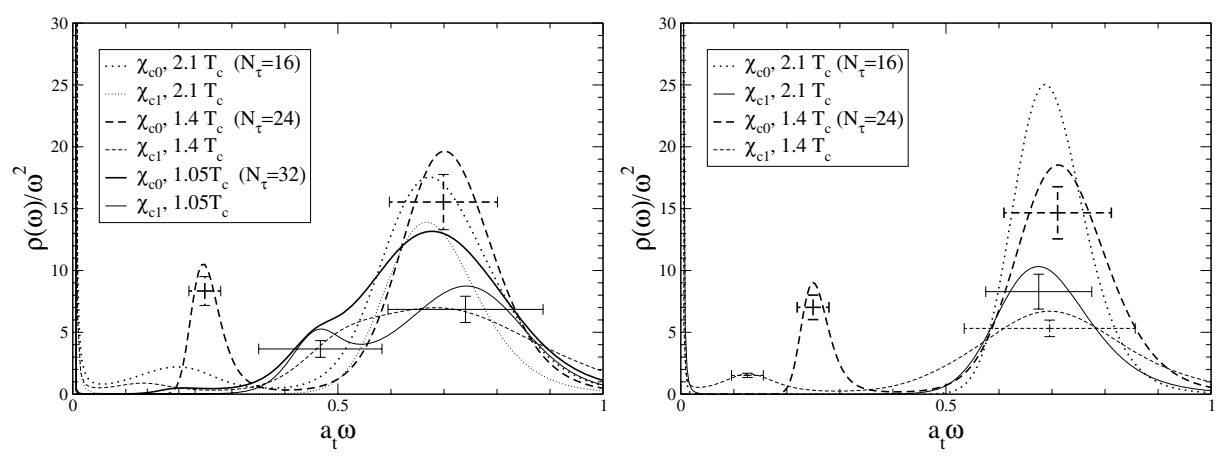

Figure 2. Spectral functions for the P-wave charmonium states $\chi_{c 0}$ and $\chi_{c 1}$, for $a_{t} m_{0}=$ 0.080 (left) and 0.092 (right). Thick lines correspond to $\chi_{c 0}$, thin lines to $\chi_{c 1}$.

remains a longer-term prospect.

\section{Acknowledgments}

This work was supported by the IITAC project, funded by the Irish Higher Education Authority under PRTLI cycle 3 of the National Development Plan and funded by IRCSET award SC/03/393Y, SFI grants 04/BRG/P0266 and 04/BRG/P0275. We are grateful to the Trinity Centre for High-Performance Computing for their support.

\section{REFERENCES}

1. T. Matsui and H. Satz, Phys. Lett. B178 (1986) 416.

2. M. Asakawa, T. Hatsuda and Y. Nakahara, Prog. Part. Nucl. Phys. 46 (2001) 459 [hep-lat/0011040].

3. M. Asakawa and T. Hatsuda, Phys. Rev. Lett. 92 (2004) 012001 [hep-lat/0308034].

4. S. Datta et al., Phys. Rev. D69 (2004) 094507 [hep-lat/0312037].

5. T. Umeda, K. Nomura and H. Matsufuru, Eur. Phys. J. C39S1 (2005) 9 [heplat/0211003].

6. R. Morrin et al., Phys. Rev. D74 (2006) 014505 [hep-lat/0604021].

7. R. Morrin et al., PoS LAT2005 (2005) 176 [hep-lat/0509115].

8. G. Aarts et al., Nucl. Phys. Proc. Suppl. 153 (2006) 296 [hep-lat/0511028].

9. C. Morningstar and M.J. Peardon, Nucl. Phys. Proc. Suppl. 83 (2000) 887 [heplat/9911003].

10. TrinLat, J. Foley et al., Phys. Rev. D73 (2006) 014514 [hep-lat/0405030].

11. C. Morningstar and M.J. Peardon, Phys. Rev. D69 (2004) 054501 [hep-lat/0311018].

12. J. Foley et al., Comp. Phys. Commun. 172 (2005) 145 [hep-lat/0505023].

13. R.K. Bryan, Eur. Biophys. J. 18 (1990) 165. 